\title{
Cirurgia estética e funcional do umbigo: técnica de plicatura transumbilical
}

\author{
Aesthetic and functional surgery of umbilicus: transumbilicous plication technique
}

\author{
Chang Yung Chia ${ }^{1}$ \\ Ana Cláudia Weck Roxo² \\ LuCIANA LABANCA ${ }^{3}$ \\ Patrícia Durgante RitTer ${ }^{3}$
}

Trabalho realizado na clínica particular do autor, Rio de Janeiro,

RJ, Brasil.

Artigo submetido pelo SGP (Sistema de Gestão de Publicações) da RBCP.

Artigo recebido: $4 / 3 / 2011$ Artigo aceito: 8/6/2011

\begin{abstract}
RESUMO
Introdução: $\mathrm{O}$ umbigo é fundamental na estética do abdome, o formato vertical, alongado e fundo é desejado pelas pacientes, entretanto, nas cirurgias estéticas abdominais, a região umbilical e peri-umbilical frequentemente é negligenciada, deixando uma lacuna sem plicatura aponeurótica e sem estreitamento do anel umbilical, resultando em um umbigo alargado e "raso". Objetivo: Os autores apresentam uma técnica de tratamento estético e funcional do umbigo, e avaliam a sua eficácia e, principalmente, a sua segurança. Método: A técnica consiste na abordagem do umbigo com uma sutura em "U", transumbilical, que aproxima a aponeurose, fecha o anel umbilical e aprofunda a sua base. $O$ estudo é retrospectivo do período de um ano, com 30 pacientes de sexo feminino, com idade entre 26 e 59 anos, com média de 42,1 anos, todas submetidas à abdominoplastia, exceto uma na qual foi corrigido isoladamente o umbigo inestético. Resultados: Todas as pacientes ficaram satisfeitas com o tamanho e o formato estético do umbigo, não houve recidiva da diástase da aponeurose nem da hérnia umbilical, não houve complicações isquêmicas, nem de contraturas cicatriciais. Conclusão: A técnica é simples, eficaz no tratamento funcional e estético, e a sutura transumbilical provou ser segura, sem complicações isquêmicas.
\end{abstract}

Descritores: Umbigo. Abdome/cirurgia. Cirurgia Plástica/métodos.

\begin{abstract}
Introduction: The umbilicus is essential for the aesthetic appearance of the abdomen, the vertical, long and deep shape is desired by the patients, however, in traditional aesthetic abdominal surgery, the rectus sheath around umbilicus is not plicated, the ring of umbilicus is not reduced, staying a gap of weakness, and the navel is widened and flat. Objective: The authors present a technique for aesthetic and functional repair of umbilicus, its efficacy and specially the safety, are analyzed. Methods: The technique consists in approach the umbilicus with a " $U$ ' shape suture across the umbilicus, plicating the rectus sheath, reducing the ring of the umbilicus, and deepening its base. It's made a retrospective study of 30 female patients, with the age between 26 and 59 years, media of 42.1 years, in the period of one year, all of them were submitted a abdominoplasty, except one which is made treatment of isolated diastasis and hernia. Results: All the patients were satisfied about the size and the shape of the umbilicus, there is no recurrence of diastasis or hernias, no ischemic complications was presented, nor scars contractions. Conclusions: The technique is simple, efficient with good functional and aesthetic results, and overall safe, without ischemic complications.
\end{abstract}

Keywords: Umbilicus. Abdomen/surgery. Plastic surgery/methods.

1. Membro Titular da Sociedade Brasileira de Cirurgia Plástica; Cirurgião do Serviço de Microcirurgia Reconstrutiva do Hospital Federal dos Servidores do Estado do Rio de Janeiro; Coordenador da Residência e Coordenador de Microcirurgia e do Centro de Estudos e de Pesquisas do Serviço de Cirurgia Plástica do Hospital Federal do Andaraí do Rio de Janeiro, Rio de Janeiro, RJ, Brasil.

2. Membro Especialista da SBCP; Cirurgiã do Serviço de Cirurgia Plástica da Universidade Estadual do Rio de Janeiro; Cirurgiã do Serviço de Cirurgia Plástica do Hospital Federal do Andaraí do Rio de Janeiro, Rio de Janeiro, RJ, Brasil.

3. Membro Especialista da SBCP; Membro da Sociedade Brasileira de Laser, Cirurgiã Plástica, Rio de Janeiro, RJ, Brasil. 


\section{INTRODUÇÃO}

O umbigo é essencial na aparência estética do abdome. Estudos recentes têm demonstrado que mulheres jovens e magras com abdomes atraentes tendem a ter umbigos pequenos e orientados verticalmente ${ }^{1-3}$ (Figura 1). Variações expressivas dovolume abdominal, de origem ponderal ou por gravidez, podem levar à flacidez, ao acúmulo de excesso cutâneo, cobrindo parcialmente o umbigo, e à diástase dos músculos reto abdominais, frequentemente acompanhada de hérnias, resultando em um umbigo alargado, raso e com o fundo plano ou abaulado (Figura 2).

A abdominoplastia é a melhor oportunidade de restaurar ou de confeccionar um umbigo mais estético, com retirada do excesso de pele, mudança do formato, correção da hérnia quando presente, estreitar o anel umbilical quando alargado, aprofundar a base do umbigo, e aproximar as bordas mediais dos músculos reto abdominais. Entretanto, nas técnicas descritas ${ }^{4}$, a plicatura não é feita na região umbilical, provavelmente para se evitar a isquemia por "estrangulamento" do mesmo, deixando, assim, uma lacuna de fragilidade que muitas vezes pode deixar visível um abaulamento nesta região, no pós-operatório. O umbigo geralmente é fixado na aponeurose nos seus pontos cardinais, podendo o fundo permanecer alargado ou abaulado, sem o formato de cone invertido.

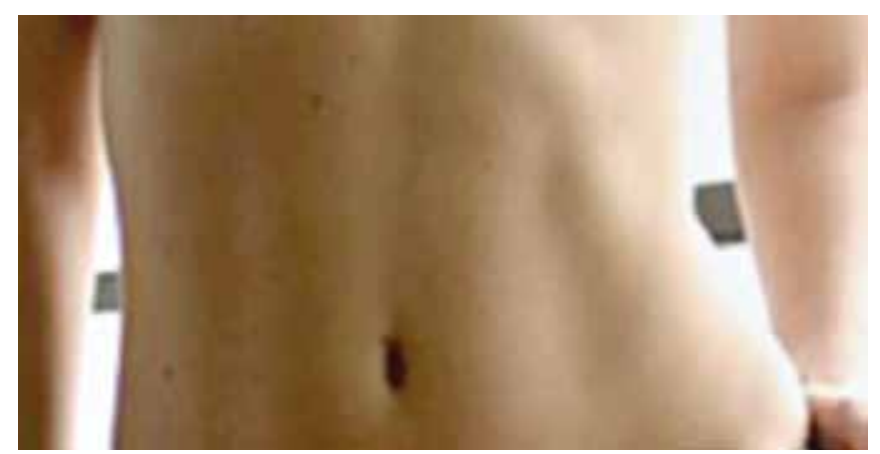

Figura 1 - Características estéticas do umbigo: alongado, vertical e fundo invisivel.

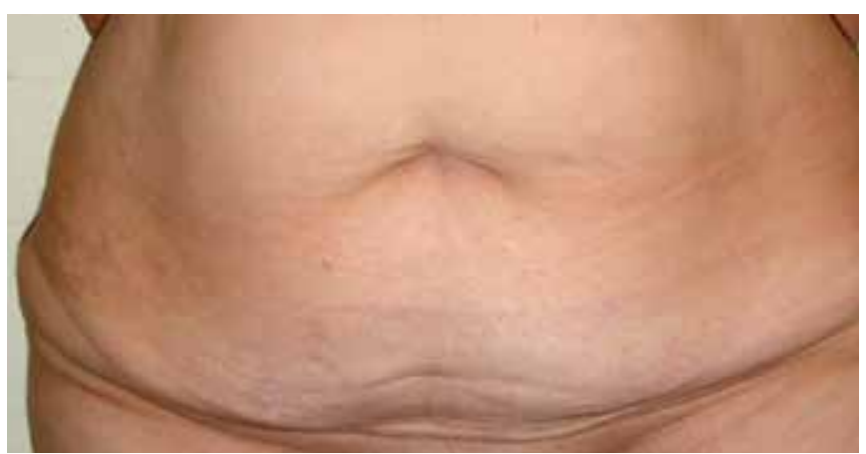

Figura 2 - Umbigo com excesso cutâneo, diástase dos músculos reto abdominais e hérnia umbilical. Note a dobra de pele, o alargamento do umbigo e fundo plano ou abaulado.
O presente trabalho propõe uma técnica simples de correção funcional e estética do umbigo, com uma sutura de plicatura transumbilical da aponeurose, "invagina" o centro do umbigo, estreitando o mesmo, e fecha o anel umbilical. Os resultados são apresentados, a eficácia e a segurança do método são analisadas.

\section{MÉTODO}

No período de um ano, a técnica foi aplicada em 30 pacientes, todos do sexo feminino (100\%), com idade entre 26 e 59 anos, média de 42,1 anos. Aidade das pacientes e as cirurgias realizadas estão discriminadas na Tabela 1.

\begin{tabular}{|c|c|c|c|c|}
\hline No & Paciente & $\mathbf{S}$ & $\mathbf{I}$ & Procedimento \\
\hline 1 & C. C. & $\mathrm{F}$ & 42 & $\begin{array}{l}\text { Abdominoplastia, lipoaspiração e } \\
\text { mamoplastia }\end{array}$ \\
\hline 2 & C. G. P. & $\mathrm{F}$ & 39 & Abdominoplastia \\
\hline 3 & F. P. & $\mathrm{F}$ & 32 & Abdominoplastia \\
\hline 4 & Z. A. & $\mathrm{F}$ & 56 & Abdominoplastia \\
\hline 5 & R. M. & $\mathrm{F}$ & 43 & Abdominoplastia e perineoplastia \\
\hline 6 & D. M. & $\mathrm{F}$ & 39 & $\begin{array}{l}\text { Abdominoplastia, HTA e mamoplastia } \\
\text { de aumento }\end{array}$ \\
\hline 7 & L. B. & $\mathrm{F}$ & 45 & Abdominoplastia, lipoaspiração e HTA \\
\hline 8 & M. Q. & $\mathrm{F}$ & 36 & Abdominoplastia e lipoaspiração \\
\hline 9 & J. C. & $\mathrm{F}$ & 47 & Abdominoplastia e lipoaspiração \\
\hline 10 & D. A. & $\mathrm{F}$ & 38 & Abdominoplastia e rinoplastia \\
\hline 11 & A. M. L. & $\mathrm{F}$ & 48 & Abdominoplastia HTA e mamoplastia \\
\hline 12 & M. C. & $\mathrm{F}$ & 43 & Abdominoplastia \\
\hline 13 & M. C. & $\mathrm{F}$ & 46 & Abdominoplastia \\
\hline 14 & J. R. & $\mathrm{F}$ & 59 & Abdominoplastia e mamoplastia \\
\hline 15 & A. L. K. & $\mathrm{F}$ & 41 & Abdominoplastia e HTA \\
\hline 16 & M. A. & $\mathrm{F}$ & 42 & $\begin{array}{l}\text { Abdominoplastia, lipoaspiração e } \\
\text { mamoplastia }\end{array}$ \\
\hline 17 & A. F. & $\mathrm{F}$ & 32 & Abdominoplastia e lipoaspiração \\
\hline 18 & C. F. & $\mathrm{F}$ & 31 & $\begin{array}{l}\text { Abdominoplastia e mamoplastia de } \\
\text { aumento }\end{array}$ \\
\hline 19 & A. L. A. & $\mathrm{F}$ & 34 & Abdominoplastia e lipoaspiração \\
\hline 20 & S. G. M. & $\mathrm{F}$ & 55 & Abdominoplastia e blefaroplastia \\
\hline 21 & V. L. & $\mathrm{F}$ & 49 & Lipoabdominoplastia \\
\hline 22 & D. C. & $\mathrm{F}$ & 58 & Abdominoplastia e lipoaspiração \\
\hline 23 & C. G. & $\mathrm{F}$ & 40 & Lipoabdominoplastia \\
\hline 24 & M. C. B. & $\mathrm{F}$ & 39 & Lipoabdominoplastia \\
\hline 25 & R. N. & $\mathrm{F}$ & 46 & Lipoabdominoplastia \\
\hline 26 & L. F. & $\mathrm{F}$ & 36 & Abdominoplastia e lipoaspiração \\
\hline 27 & T. M. O. & $\mathrm{F}$ & 53 & $\begin{array}{l}\text { Abdominoplastia e mastopexia com } \\
\text { implante }\end{array}$ \\
\hline 28 & D. R. & $\mathrm{F}$ & 26 & Correção da diástase e da hérnia umbilical \\
\hline 29 & E. A. A. & $\mathrm{F}$ & 39 & $\begin{array}{l}\text { Abdominoplastia, lipoaspiração e } \\
\text { mamoplastia }\end{array}$ \\
\hline 30 & D. F. & F & 29 & Abdominoplastia e lipoaspiração \\
\hline
\end{tabular}




\section{Técnica Cirúrgica}

A técnica pode ser executada em abdominoplastias, lipoabdominoplastias, miniabdominoplastias ou em correções isoladas da diástase e hérnias primárias ou secundárias.

Após a dissecação do retalho cutâneo-adiposo abdominal, a diástase dos músculos reto abdominais é identificada e marcada. Na presença da hérnia umbilical (Figuras 3 e 4A), o anel herniário é dissecado, o conteúdo reduzido e a aponeurose fechada com fio inabsorvível.

A plicatura do abdome é feita com fio de polipropileno 0-0, com pontos simples ou cruzados, do apêndice xifoide até o púbis, o mais próximo possível do pedículo umbilical, cranial e caudalmente. $\mathrm{O}$ tratamento da região umbilical é feito com fio de nylon monofilamentar 2.0, com agulha de 3 ou $4 \mathrm{~cm}$. Passa-se a agulha na aponeurose de um dos lados do umbigo, entrando lateralmente à marcação da diástase e saindo nesta (Figura 4B). Em seguida, a agulha entra na base do pedículo umbilical e se dirige superficialmente, "raspando" o fundo do umbigo e desce novamente transfixando a base do pedículo do outro lado. No outro lado, a agulha entra na aponeurose, na marcação da diástase e sai lateral à mesma. $\mathrm{O}$ mesmo fio volta, alguns milímetros mais caudal, repetindo o mesmo trajeto até retornar ao início (Figura 4C), onde o nó é feito, com tensão suficiente de aproximar as bordas do músculo reto abdominal, sem estrangular o pedículo umbilical, configurando um ponto em "U". Este ponto aproxima a aponeurose, corrigindo a frequente falha deixada nesta região e aprofunda o umbigo (Figura 4D).

Em abdominoplastia, a incisão no retalho para confecção do umbigo é feita com ressecção de um segmento cutâneo de aproximadamente de $2 \mathrm{~cm}$ no sentido longitudinal e de 2 a 3 $\mathrm{mm}$ de largura, acompanhada de uma quantidade limitada de tecido subcutâneo (Figura 5). A sutura do umbigo é feita com fio nylon monofilamentar 5-0 com pontos de "Gilles".

No tratamento do umbigo alargado, sem abdominoplastia, após a aproximação da aponeurose e fixação do umbigo, o tecido adiposo subcutâneo dever ser aproximado fixando a fáscia de Scarpa na base umbilical bilateralmente, para dar mais profundidade e tornar a fenda umbilical estreita (Figuras 4E e 4F).
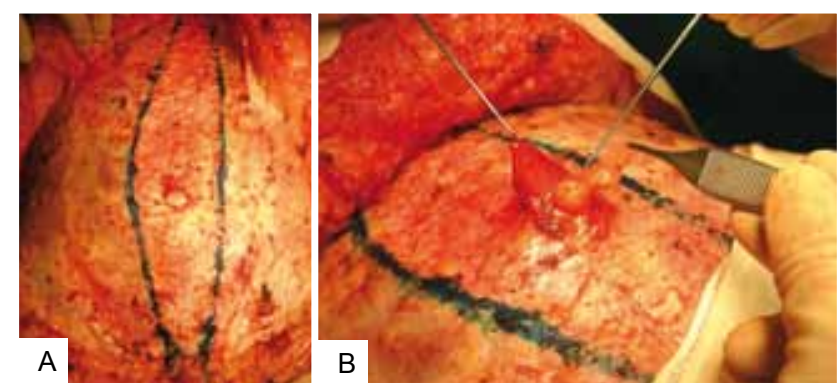

Figura 3 - A: Per-operatório de abdominoplastia estética com diástase dos músculos reto abdominais e hérnia umbilical; $\boldsymbol{B}$ : Detalhe da hérnia umbilical.

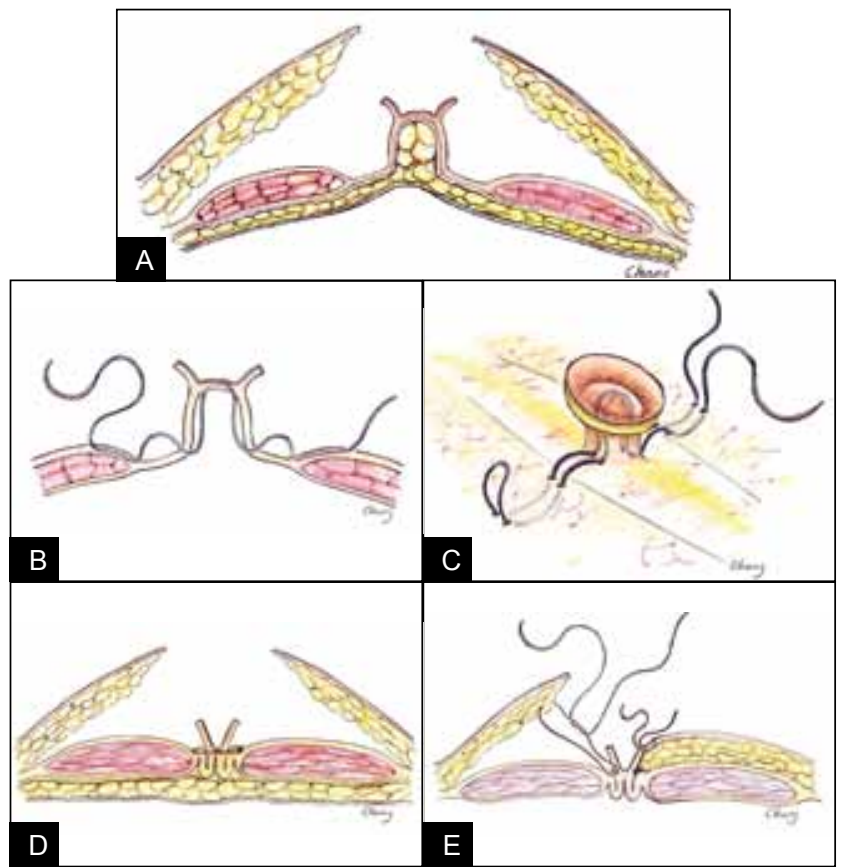

Figura 4-A: Diástase dos músculos retos abdominais e hérnia umbilical; B: Plicatura da aponeurose a nível umbilical, interessando o anel herniário, o fundo umbilical e a aponeurose

bilateral; C: Vista oblíqua da sutura tipo "U”; D: Síntese,

sem tensão, aproximando os músculos retos, fechando o anel herniário e aprofundamento da base umbilical; $\boldsymbol{E}$ : Aproximação do retalho cutâneo com fixação da fáscia de Scarpa na base lateral do umbigo.

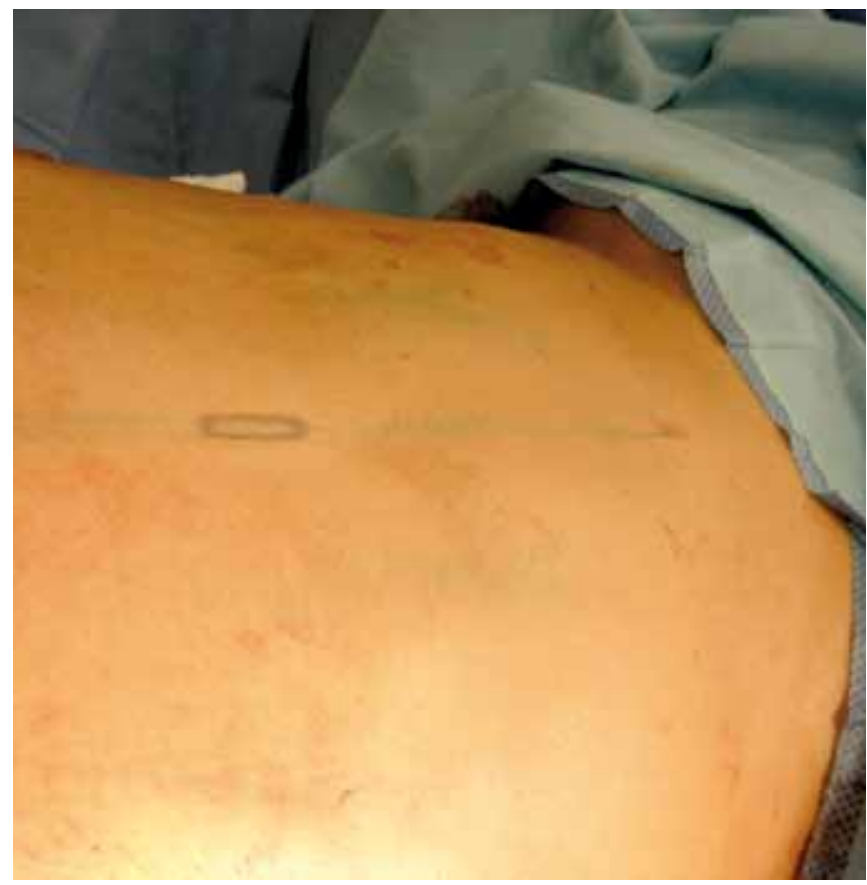

Figura 5 - Marcação do umbigo no retalho abdominal. 


\section{RESULTADOS}

Os umbigos tratados apresentaram-se estreitos, alongados verticalmente e profundos. As hérnias foram eficientemente tratadas.

Não foram observadas complicações, como necrose, deiscência de sutura ou contração cicatricial e constrição umbilical (Figuras 6 a 10).

Não houve recidiva da diástase da aponeurose, nem da hérnia umbilical.
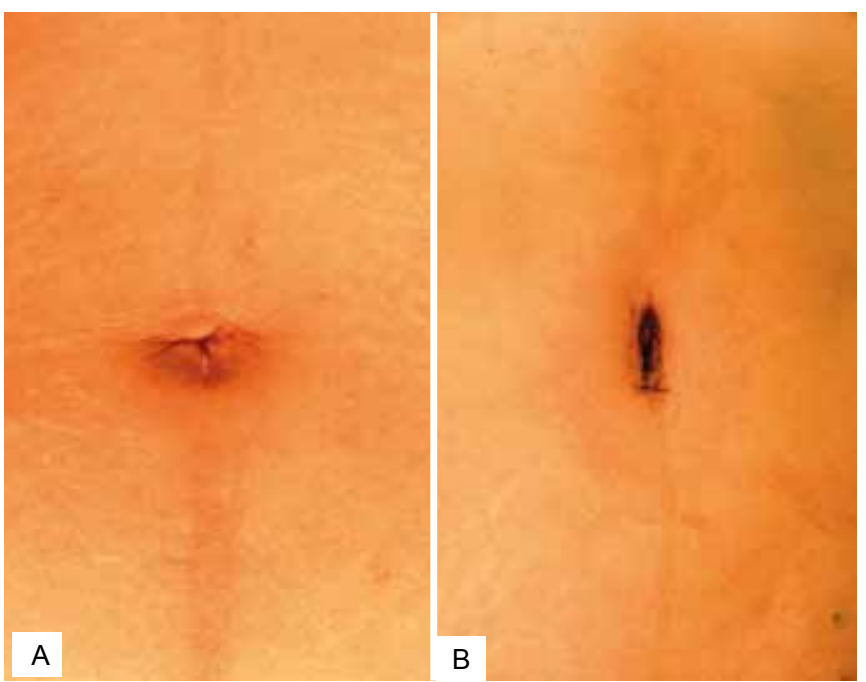

Figura 6-A: Pré-operatório. B: Pós-operatório imediato.
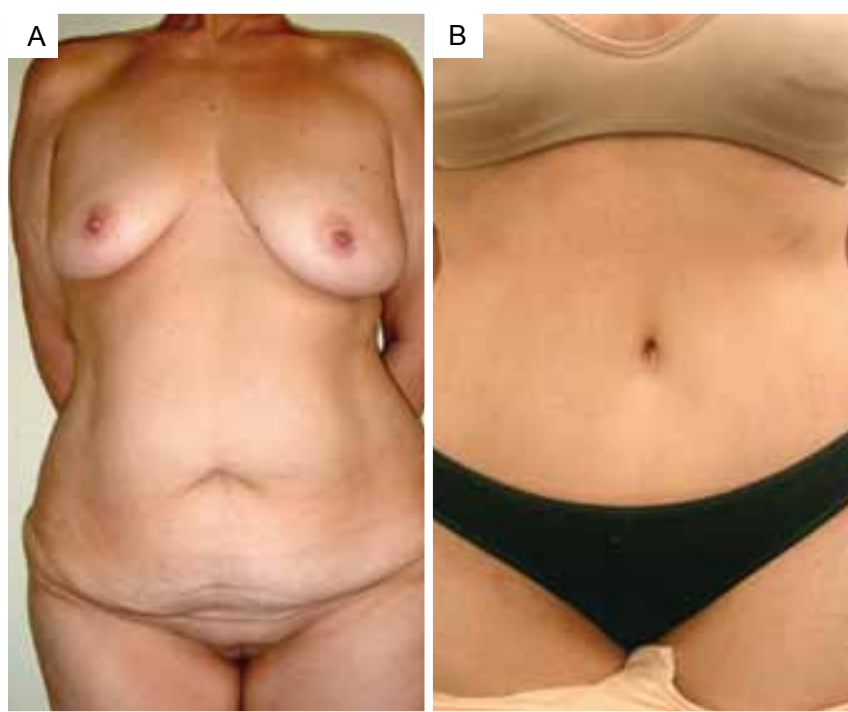

Figura 7-A: Pré-operatório. B: Pós-operatório de abdominoplastia e mastopexia com implante mamário, herniorrafia umbilical, e plicatura da aponeurose dos músculos reto abdominais "transumbilical".
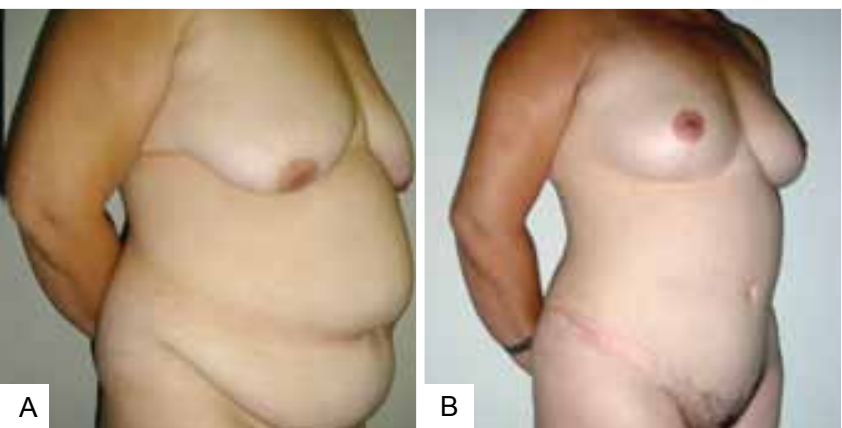

Figura 8-A: Pré-operatório. B: Pós-operatório de mastopexia, abdominoplastia com plicatura da aponeurose dos músculos reto abdominais e fixação transumbilical.
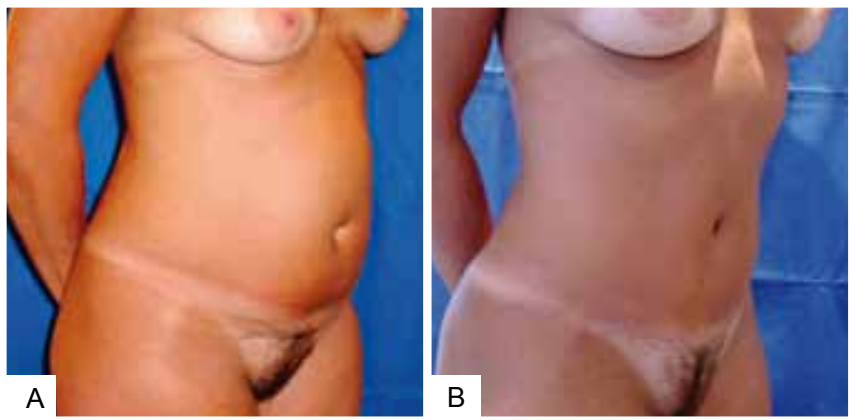

Figura 9-A: Pré-operatório. B: Pós-operatório de abdominoplastia e plicatura transumbilical.
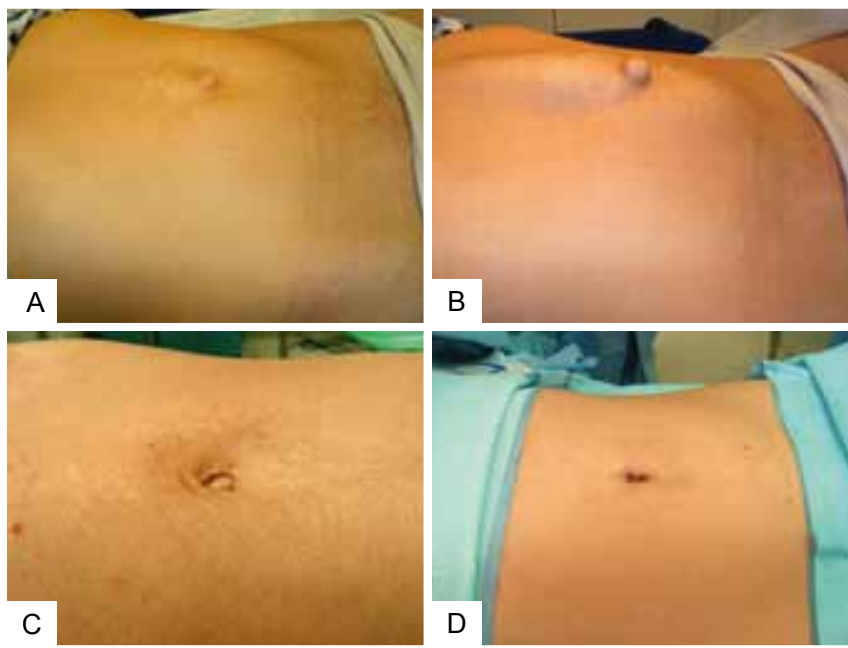

Figura 10-A: Paciente com grave diástase dos músculos reto abdominais e hérnia umbilical, em decúbito dorsal e em repouso; B: Paciente com mínimo de esforço de prensa abdominal evidenciando incompetencia importante; $\boldsymbol{C}$ : Pós-operatório da correção da hérnia umbilical e diástase dos músculos reto abdominais sem a sutura de plicatura transumbilical; D: Pósoperatório imediato da reoperação com acesso peri-umbilical para confecção da plicatura e fixação transumbilical. 


\section{DISCUSSÃO}

$\mathrm{O}$ umbigo normalmente tem o formato de um cone invertido, alongado verticalmente e com fundo não visível. Ele é o primeiro a denunciar o excesso de pele ou de peso no abdome.

Na presença de diástase dos músculos reto abdominais, o umbigo alarga-se no sentido horizontal e o fundo tornase aparente. Hérnias, que com frequência acompanham diástase, deformam ainda mais a única cicatriz normal do corpo humano ${ }^{5}$.

As pacientes têm uma preocupação especial em relação ao umbigo, por este constituir a unidade estética central do abdome. Várias técnicas de onfaloplastias têm sido descritas visando à confecção de um umbigo de aspecto natural, afastando o estigma da cirurgia ${ }^{5-7}$, outras tentam descrever o local exato que o mesmo deva ser fixado ${ }^{8}$, todavia nenhuma delas propõe o tratamento funcional desta região.

É na abdominoplastia que as pacientes têm a melhor chance da correção estética e funcional do umbigo, e, para isto são necessários: a exérese da redundância cutânea, o tratamento das frequentes hérnias, a aproximação transumbilical dos músculos reto abdominais, o estreitamento do anel umbilical, o aprofundamento da base do umbigo no nível da aponeurose, a preservação da gordura periumbilical e a confecção de um formato longo e estreito do orifício do neoumbigo no retalho abdominal.

\section{CONCLUSÃO}

A técnica é de fácil execução, eficiente no tratamento tanto do umbigo alargado e raso, quanto da diástase peri-umbilical, $\mathrm{e}$, das hérnias frequentemente presentes. A aparência estética atingiu as expectativas das pacientes. Sobretudo, comprovouse que a sutura de plicatura em "U" transfixante no pedículo umbilical é segura, sem complicações isquêmicas.

\section{REFERÊNCIAS}

1. Choudhary S, Taams KO. Umbilicosculpture: a concept revisited. $\mathrm{Br}$ J Plast Surg. 1998;51(7):538-41.

2. Craig SB, Faller MS, Puckett CL. In search of the ideal female umbilicus. Plast Reconstr Surg. 2000;105(1):389-92.

3. Lee MJ, Mustoe TA. Simplified technique for creating a youthful umbilicus in abdominoplasty. Plast Reconstr Surg. 2002;109(6):2136-40.

4. Chang TN, Baroudi R. Abdominoplasty techniques. In: Mathes SJ, ed. Plastic surgery: trunk and lower extremity. Vol. 6. 2nd ed. New York:Saunders Elsevier;2006. p.119-91

5. Niranjan NS, Sataiano JJ. An anatomical method for re-siting the umbilicus. Plast Reconstr Surg. 2004;113(7):2194-8.

6. Rozen SM, Redett R. The two-dermal-flap umbilical transposition: a natural and aesthetic umbilicus after abdominoplasty. Plast Recontr Surg. 2007;119(7):2255-62.

7. Juri J, Juri C, Raiden G. Reconstruction of the umbilicus in abdominoplasty. Plast Reconstr Surg. 1979;63(4):580-2.

8. Abhyankar SV, Rajguru AG, Patil PA. Anatomical localization of the umbilicus: an Indian study. Plast Reconstr Surg. 2006;117(4):1153-7.

9. Chang YC. Cirurgia estética e funcional do umbigo: uma técnica simples. In: $45^{\circ}$ Congresso Brasileiro de Cirurgia Plástica; 2008 Nov 12 - 15; Brasília, Brasil.

\section{Correspondência para:}

Chang Yung Chia

Av. Lúcio Costa, 3360, bloco 8, apto. 2402 - Barra da Tijuca - Rio de Janeiro, RJ, Brasil - CEP 22630-010

E-mail: changplastica@gmail.com 